\title{
Nonlinear observer for vehicle velocity estimation
}

\author{
Lars Imsland, Tor A. Johansen* and Thor I. Fossen* \\ SINTEF ICT, Applied Cybernetics, N-7465 Trondheim, Norway \\ Jens C. Kalkkuhl and Avshalom Suissa \\ DaimlerChrysler Research and Technology, 70456 Stuttgart, Germany
}

Copyright (C) 2006 SAE International

\begin{abstract}
A nonlinear observer for estimation of lateral and longitudinal velocity of automotive vehicles is proposed, based on acceleration and yaw rate measurements in addition to wheel speed and steering angle measurements. Stability of the observer is proved in the form of input-to-state stability of the observer error dynamics, under an assumption on the friction model. This assumption is treated with some detail. The observers are validated on experimental data from cars.
\end{abstract}

\section{INTRODUCTION}

Automotive feedback control systems for vehicle handling and/or active safety usually depend on information of vehicle velocity, sometimes in the form of the vehicle body side slip angle. While concepts for direct measurement of velocity exist, they are generally considered too expensive for use in production cars. Hence, concepts for inferential estimation of vehicle velocity using other (cheaper) measurements are of considerable interest.

The main goal of this work is to develop a nonlinear observer for estimation of vehicle velocity. To provide a theoretical foundation for its implementation, explicit stability conditions for convergence of the estimated states are analyzed. The observer is based on nonlinear models for taking the nonlinear dynamics (mainly due to highly nonlinear friction and Coriolis forces) into account, and to obtain simple designs with few tuning knobs (as opposed to Extended Kalman Filter (EKF) designs). Another significant advantage over EKF designs, is that real-time solution of the Riccati differential equations is avoided, such that the observer can be implemented more efficiently in a low-cost embedded computer unit.

A non-linear friction model is used for better exploiting the measurements. An important parameter in many friction

${ }^{*}$ Also affiliated with NTNU, Department of Engineering Cybernetics, N-7491 Trondheim, Norway models, the maximal friction coefficient $\mu_{H}$, is known to vary significantly with different road conditions. We will assume this parameter to be known, or estimated by other means. While simultaneous estimation of velocity and $\mu_{H}$ might be a feasible path, we argue that estimation of $\mu_{H}$ requires special attention depending on the application the observer is used for, since it will be only weakly observable for many (normal) driving conditions, requiring monitoring, resetting and other logic functions to be implemented (see e.g. [7]).

Earlier work on observers for estimation of lateral velocity are mainly based on linear or quasi-linear techniques, e.g. $[3,19,17,2]$. A nonlinear observer linearizing the observer error dynamics have been proposed in $[9,10]$. The same type of observer, in addition to an observer based on forcing the dynamics of the nonlinear estimation error to the dynamics of a linear reference system, are investigated in [5]. The problem formulation there assumes that the longitudinal wheel forces are known, as the observer implemented in ESP also does [18]. In our work, we do not make this assumption, as such information is not always available.

The Extended Kalman Filter (EKF) is used as a nonlinear observer for estimating vehicle velocity and tyre forces in $[13,14]$, thus without the explicit use of friction models. A similar, but simpler, approach is suggested in [2]. An EKF based on a tyre-road friction models that also included estimation of the adhesion coefficient and road inclination angle is suggested in [16]. In [1], the use of an EKF is considered, based on a nonlinear tyre-friction model, that also includes estimation of cornering stiffness. The strategy proposed in [11] combines dynamic and kinematic models of the vehicle with numerical bandlimited integration of the equations to provide a side-slip estimate. In [4] the side-slip angle is estimated along with yaw rate in an approach that has similarities with the one considered herein, but without yaw-rate measurements. The approach is validated using experimental data, but there are no stability proofs. 
In [6], we approached this estimation problem using modular observers. In the present paper, a single combined observer is proposed, with the advantage (compared to the approach in [6]) that Coriolis forces are fully taken into account in estimation of longitudinal velocity. Moreover, robustness to friction model errors are disussed and the regional convergence of the observer error is expanded with global boundedness of the observer state.

\section{VEHICLE MODELING}

The geometry of the vehicle is illustrated in Figure 1. The vehicle velocity is defined in a body-fixed coordinate system with the origin at the vehicle center of gravity (CG, assumed constant), with $x$-axis pointing forward and $y$-axis to the left. There is also a coordinate system in the center of each wheel, aligned with the orientation of the wheel. The distance from CG to each wheel center is denoted $h_{i}$, with $i$ being wheel index. Together with the angles $\gamma_{i}$, this define the vehicle geometry. Neglecting suspension

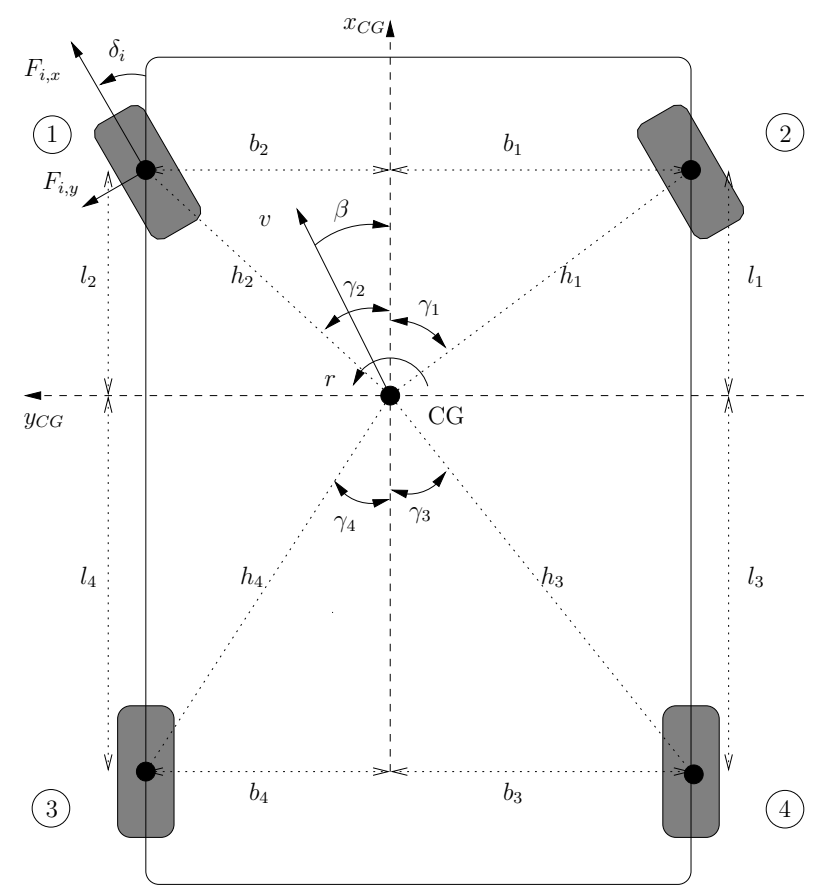

Figure 1: Horizontal axis systems, geometric definitions, wheel forces, speed, slip angle and yaw rate.

dynamics, we will assume that we can consider the vehicle a rigid body, for which the rigid body dynamics (with respect to the CG coordinate system) can be written

$$
\mathbf{M} \dot{\nu}+\mathbf{C}(\boldsymbol{\nu}) \boldsymbol{\nu}=\boldsymbol{\tau}
$$

where $\nu$ is a vector containing the body generalized velocities. The matrices $\mathbf{M}$ and $\mathbf{C}$ are the inertia, and Coriolis and centripetal matrices, respectively. The vector $\tau$ consists of forces and torques on the vehicle, mainly friction forces acting via the wheels, but also gravitational and aerodynamic (wind and air resistance) forces are at work.
By making the following assumptions,

- only include motion in the plane (ignore dynamics related to vertical motion, including roll and pitch),

- ignore effect of caster and camber,

- only include tyre friction forces,

the vehicle dynamics are described by longitudinal velocity $v_{x}$, lateral velocity $v_{y}$ and yaw rate $r$, resulting in the "two-track model" [10], with

$$
\mathbf{M}=\left(\begin{array}{ccc}
m & 0 & 0 \\
0 & m & 0 \\
0 & 0 & J_{z}
\end{array}\right), \quad \mathbf{C}(\boldsymbol{\nu})=\left(\begin{array}{ccc}
0 & -m r & 0 \\
m r & 0 & 0 \\
0 & 0 & 0
\end{array}\right)
$$

The generalized forces $\tau=\left(f_{x}, f_{y}, \tau_{z}\right)^{\top}$ are forces and torque generated by friction between the wheels and the ground,

$$
\boldsymbol{\tau}=\sum_{i=1}^{4}\left(\begin{array}{c}
\mathbf{I}_{2 \times 2} \\
\mathbf{g}_{i}^{\top}
\end{array}\right) \mathbf{R}\left(\delta_{i}\right) \mathbf{F}_{i} .
$$

The friction forces $\mathbf{F}_{i}$ working at each wheel (see Figure 1) are functions of velocity difference between vehicle and tyres, see below. They are transformed from the wheel coordinate systems to CG:

- The forces generated by the tyres in body-fixed coordinates for each wheel $i$, are:

$$
\mathbf{f}_{i}=\left(f_{i, x}, f_{i, y}\right)^{\top}=\mathbf{R}\left(\delta_{i}\right) \mathbf{F}_{i}
$$

where $\mathbf{F}_{i}=\left(F_{i, x}, F_{i, y}\right)$ are the forces acting on the wheel in the wheel-fixed coordinate system. The rotation matrix induced by the steering angle $\delta_{i}$ is

$$
\mathbf{R}\left(\delta_{i}\right)=\left(\begin{array}{cc}
\cos \delta_{i} & -\sin \delta_{i} \\
\sin \delta_{i} & \cos \delta_{i}
\end{array}\right) .
$$

- For the torque, it is convenient to define the geometry vector

$$
\mathbf{g}_{i}=\left(\begin{array}{c}
-h_{i} \sin \psi_{i} \\
h_{i} \cos \psi_{i}
\end{array}\right)
$$

where the angles $\psi_{i}$ are introduced to get a uniform representation, $\psi_{1}=-\gamma_{1}, \psi_{2}=\gamma_{2}, \psi_{3}=\pi+\gamma_{3}$ and $\psi_{4}=\pi-\gamma_{4}$. The generated torque about the vertical axis through the $C G$ is then for each wheel

$$
\tau_{i, z}=\mathbf{g}_{i}^{\top} \mathbf{f}_{i} .
$$

In most friction models, the friction forces are functions of tyre slips, $\mathbf{F}_{i}=\mathbf{F}_{i}\left(\lambda_{i, x}, \lambda_{i, y}\right)$, where the slips $\lambda_{i, x}$ and $\lambda_{i, y}$ are measures of the relative difference in vehicle and tyre longitudinal and lateral velocity for wheel $i$. The definitions for tyre slips we will use herein, are

$$
\lambda_{i, x}=\frac{\omega_{i} R_{d y n}-V_{i, x}}{V_{i, x}}, \quad \lambda_{i, y}=\sin \alpha_{i},
$$


where $\omega_{i}$ is the wheel angular velocity and $R_{d y n}$ is the dynamic wheel radius, and the tyre slip angles are calculated as

$$
\alpha_{i}=\delta_{i}-\arctan \frac{v_{i, y}}{v_{i, x}}
$$

and $V_{i, x}$ is the velocity in $x$-direction in the wheel coordinate system,

$$
V_{i, x}=\sqrt{v_{i, x}^{2}+v_{i, y}^{2}} \cos \alpha_{i} .
$$

The longitudinal and lateral velocities of the wheel center in the body-fixed coordinate system are $v_{i, x}=v_{x} \pm r b_{i}$ and $v_{i, y}=v_{y} \pm r l_{i}$. For the tyre slip angles to be well defined, we assume for convenience that there is no reverse motion,

Assumption $1 v_{i, x}>0$ for $i=1, \ldots, 4$.

The tyre slips depend on the vehicle states and the time-varying, measured steering angles $\boldsymbol{\delta}=\left(\delta_{1}, \ldots, \delta_{4}\right)^{\mathrm{T}}$ and wheel angular speeds $\boldsymbol{\omega}=\left(\omega_{1}, \ldots, \omega_{4}\right)^{\top}$. We will therefore use the notations $\mathbf{F}_{i}=\mathbf{F}_{i}\left(\lambda_{i, x}, \lambda_{i, y}\right)=$ $\mathbf{F}_{i}\left(v_{x}, v_{y}, r, \delta_{i}, \omega_{i}\right)$ interchangeably, depending on context.

We make the following assumption on the friction model: Assumption 2 There exist a positive constant $c_{1}$ and sets $\Delta, \Omega$ and a convex set $X(\boldsymbol{\delta}, \boldsymbol{\omega})$, such that the friction model is continuously differentiable in $\mathbf{x}=\left(v_{x}, v_{y}, r\right)^{\top}$ with $\left\|\frac{\partial \mathbf{F}_{i}\left(\mathbf{x}, \delta_{i}, \omega_{i}\right)}{\partial \mathbf{x}}\right\|$ bounded for $\mathbf{x} \in X(\boldsymbol{\delta}, \boldsymbol{\omega}), \boldsymbol{\delta} \in \Delta$ and $\boldsymbol{\omega} \in \Omega$, and

$$
\begin{aligned}
\sum_{i=1}^{4}\left(\frac{\partial F_{i, y}\left(\mathbf{x}, \delta_{i}, \omega_{i}\right)}{\partial v_{y}}\right. & \cos \delta_{i} \\
+ & \left.\frac{\partial F_{i, x}\left(\mathbf{x}, \delta_{i}, \omega_{i}\right)}{\partial v_{y}} \sin \delta_{i}\right)<-c_{1},
\end{aligned}
$$

for all $\boldsymbol{\delta} \in \Delta, \boldsymbol{\omega} \in \Omega$, and $\mathrm{x} \in X(\boldsymbol{\delta}, \boldsymbol{\omega})$.

The physical relevance of this assumption is discussed later.

Remark 1 The sets $X(\delta, \omega)$ and $\Delta$ will depend on which friction model is used. For example, using linear friction models, $X$ depends on $\delta$ only:

$$
\begin{aligned}
\Delta & =\left\{\delta_{i}:\left|\delta_{i}\right| \leq \bar{\delta}, i=1, \ldots, 4\right\}, \\
X(\boldsymbol{\delta}) & =\left\{\mathbf{x}=\left(v_{x}, v_{y}, r\right)^{\top}:\left|\alpha_{i}\right| \leq \bar{\alpha},|r| \leq \bar{r}, v_{x}>\bar{r} b_{i}\right\} .
\end{aligned}
$$

Note that $\alpha_{i}$ depends on $\delta_{i}$.

The following result regarding the friction forces holds due to Assumption 2. The proof is very similar to Lemma 1 in [6], and is therefore omitted.

Lemma 1 There exist positive constants $c_{i}, i=1, \ldots, 6$ such that for all $\mathrm{x}, \hat{\mathrm{x}} \in X(\boldsymbol{\delta}, \boldsymbol{\omega}), \boldsymbol{\delta} \in \Delta, \boldsymbol{\omega} \in \Omega$, the following holds:

$$
\begin{aligned}
& \tilde{v}_{y} \sum_{i=1}^{4}\left[\begin{array}{ll}
0 & 1]
\end{array}\right] \mathbf{R}\left(\delta_{i}\right)\left(\mathbf{F}_{i}\left(\mathbf{x}, \delta_{i}, \omega_{i}\right)-\mathbf{F}_{i}\left(\hat{\mathbf{x}}, \delta_{i}, \omega_{i}\right)\right) \\
& \leq-c_{1} \tilde{v}_{y}^{2}+c_{2}|\tilde{r}|\left|\tilde{v}_{y}\right|+c_{3}\left|\tilde{v}_{x}\right|\left|\tilde{v}_{y}\right| \\
& \frac{1}{J_{z}} \sum_{i=1}^{4} \mathbf{g}_{i}^{\top} \mathbf{R}\left(\delta_{i}\right)\left(\mathbf{F}_{i}\left(\mathbf{x}, \delta_{i}, \omega_{i}\right)-\mathbf{F}_{i}\left(\hat{\mathbf{x}}, \delta_{i}, \omega_{i}\right)\right) \\
& \leq c_{4}\left|\tilde{v}_{y}\right|+c_{5}|\tilde{r}|+c_{6}\left|\tilde{v}_{x}\right|
\end{aligned}
$$

\begin{tabular}{cl}
\hline Symbol & Measurement \\
\hline$a_{x}, a_{y}$ & Longitudinal/lateral acceleration \\
$r$ & yaw rate \\
$\omega_{i}$ & Rotational speed wheel $i$ \\
$\delta_{i}$ & Steering angle wheel $i$ \\
\hline
\end{tabular}

Table 1: Measurements

where $\left(\tilde{v}_{x}, \tilde{v}_{y}, \tilde{r}\right):=\mathbf{x}-\hat{\mathbf{x}}$.

\section{OBSERVER DESIGN}

The main variables to be estimated by the observer are the lateral velocity $v_{y}$ and the longitudinal velocity $v_{x}$. From these two, the vehicle body side slip angle (see Figure 1) can be found from $\beta=\arctan \left(-v_{y} / v_{x}\right)$. The measurements used by the observer are summarized in Table 1. Acceleration and yaw rate sensors are assumed placed in CG. The acceleration measurements are assumed to have bias removed, and are corrected for gravity components due to vehicle roll/pitch, based on suspension stiffness and assuming a flat road.

In addition to $v_{x}$ and $v_{y}$, we will use $r$ as a state to be estimated to better exploit the yaw-rate measurement. Thus, the state to be estimated is $\mathbf{x}=\left(v_{x}, v_{y}, r\right)^{\top}$. Based on the model derived in the previous section, we use the following equations as system model for the observer:

$$
\begin{aligned}
\dot{v}_{x} & =v_{y} r+a_{x} \\
\dot{v}_{y} & =-v_{x} r+a_{y} \\
\dot{r} & =\frac{1}{J_{z}} \sum_{i=1}^{4} \mathbf{g}_{i}^{\top} \mathbf{R}\left(\delta_{i}\right) \mathbf{F}_{i}\left(v_{x}, v_{y}, r, \delta_{i}, \omega_{i}\right) .
\end{aligned}
$$

We propose the following observer ${ }^{1}$ :

$$
\begin{aligned}
& \dot{\hat{v}}_{x}=\hat{v}_{y} r+a_{x}+\sum_{i=1}^{4} K_{i}\left(a_{x}, \boldsymbol{\omega}\right)\left(v_{x, i}-\hat{v}_{x}\right) \\
& \dot{\hat{v}}_{y}=-\hat{v}_{x} r+a_{y}-K_{v_{y}}\left(m a_{y}-\sum_{i=1}^{4}\left[\begin{array}{ll}
0 & 1
\end{array}\right] \mathbf{R}\left(\delta_{i}\right) \hat{\mathbf{F}}_{i}\right) \\
& \dot{\hat{r}}=\frac{1}{J_{z}} \sum_{i=1}^{4} \mathbf{g}_{i}^{\top} \mathbf{R}\left(\delta_{i}\right) \hat{\mathbf{F}}_{i}+K_{r}(r-\hat{r}),
\end{aligned}
$$

where $v_{x, i}$ is transformed measurement from wheel speed of wheel $i$ assuming zero slips,

$$
v_{x, i}=R_{d y n} \omega_{i} \cos \delta_{i} \pm b_{i} r .
$$

The gains in the longitudinal velocity equation, $K_{i}\left(a_{x}, \boldsymbol{\omega}\right)$, depend on the measurements. The tuning of these functions and the other two gains are discussed in the next section. For now we assume

\footnotetext{
${ }^{1}$ In the sequel, we sometimes use $\mathbf{F}_{i}$ for $\mathbf{F}_{i}\left(\mathbf{x}, \delta_{i}, \omega_{i}\right)$ and $\hat{\mathbf{F}}_{i}$ for $\mathbf{F}_{i}\left(\hat{\mathbf{x}}, \delta_{i}, \omega_{i}\right)$
} 
Assumption $3 K_{i}\left(a_{x}, \boldsymbol{\omega}\right)$ is piecewise continuous in time, and lower bounded, $K_{i}\left(a_{x}, \boldsymbol{\omega}\right)>k_{x}>0$.

The properties of this observer will be analyzed by examining the stability properties of the observer error, $\tilde{\mathbf{x}}=$ $\mathbf{x}-\hat{\mathbf{x}}$, where $\hat{\mathbf{x}}=\left(\hat{v}_{x}, \hat{v}_{y}, \hat{r}\right)^{\mathrm{T}}$. Before we write up the observer error dynamics, we briefly discuss the injection term to the $v_{x}$-part.

In general, the wheel slips are non-zero which means that $v_{x, i} \neq v_{x}$. If the slips were known exactly, the correct equation (for each wheel) would be

$$
v_{x}=\frac{1}{1+\lambda_{i, x}} \frac{\cos \left(\delta_{i}-\alpha_{i}\right)}{\cos \alpha_{i}} R_{d y n} \omega_{i} \pm b_{i} r .
$$

If we define $\tilde{v}_{x}=v_{x}-\hat{v}_{x}$, then we can write

$$
\begin{aligned}
\sum_{i=1}^{4} K_{i}\left(a_{x}, \boldsymbol{\omega}\right)\left(v_{x, i}-\hat{v}_{x}\right) & =\sum_{i=1}^{4} K_{i}\left(a_{x}, \boldsymbol{\omega}\right)\left(v_{x, i}-v_{x}+\tilde{v}_{x}\right) \\
& =\sum_{i=1}^{4} K_{i}\left(a_{x}, \boldsymbol{\omega}\right) \tilde{v}_{x}+u
\end{aligned}
$$

where

$u=\sum_{i=1}^{4} K_{i}\left(a_{x}, \boldsymbol{\omega}\right)\left(\cos \delta_{i}-\frac{1}{1+\lambda_{i, x}} \frac{\cos \left(\delta_{i}-\alpha_{i}\right)}{\cos \alpha_{i}}\right) R_{d y n} \omega_{i}$

is an error-term due to non-zero slip. When the slips are zero, then $u(t)=0$.

With this observation in place, the observer error dynamics can be written

$$
\begin{aligned}
& \dot{\tilde{v}}_{x}=\tilde{v}_{y} r-\sum_{i=1}^{4} K_{i}\left(a_{x}, \boldsymbol{\omega}\right) \tilde{v}_{x}+u \\
& \dot{\tilde{v}}_{y}=-\tilde{v}_{x} r+K_{v_{y}} \sum_{i=1}^{4}\left[\begin{array}{ll}
0 & 1]
\end{array}\right] \mathbf{R}\left(\delta_{i}\right)\left(\mathbf{F}_{i}-\hat{\mathbf{F}}_{i}\right) \\
& \dot{\tilde{r}}=\frac{1}{J_{z}} \sum_{i=1}^{4} \mathbf{g}_{i}^{\top} \mathbf{R}\left(\delta_{i}\right)\left(\mathbf{F}_{i}-\hat{\mathbf{F}}_{i}\right)-K_{r} \tilde{r}
\end{aligned}
$$

The stability properties we want to prove, are that when $u(t)=0$ the observer error dynamics are exponentially stable (Theorem 1). When $u(t) \neq 0$, natural properties to aim for are that the influence $u$ on the observer should not lead to divergence of the estimate, when $u$ is small the influence on the estimate should be small, and that when $u$ vanishes, the estimation error should go to zero. These properties correspond to input-to-state stability (ISS) [15, 8], characterized by convergence of zero-input response, and that the zero state response is bounded for bounded input. ISS with $u(t)$ as input will be shown in Corollary 1.

For initial states in the set $X_{s}(\rho ; \boldsymbol{\delta}, \boldsymbol{\omega}) \subset X(\boldsymbol{\delta}, \boldsymbol{\omega})$, Theorem 1 guarantees exponential convergence for initial observer error $\|\tilde{\mathbf{x}}(0)\| \leq \rho$. The set is formally defined as $X_{s}(\rho ; \boldsymbol{\delta}, \boldsymbol{\omega})=\{\mathbf{x}: B(\mathbf{x}, \rho)\}$ where $B(\mathbf{x}, \rho):=\{\mathbf{z}$ : $\|\mathbf{z}-\mathbf{x}\| \leq \rho\}$ is the ball of radius $\rho$ around $\mathbf{x}$.
Theorem 1 Assume that $\rho$ and $\Delta, \Omega$ are such that $\mathbf{x}(t) \in$ $X_{s}(\rho ; \boldsymbol{\delta}, \boldsymbol{\omega}), \forall(\boldsymbol{\delta}, \boldsymbol{\omega}) \in \Delta \times \Omega, \forall t \geq 0$, and that $u(t)=0$, $\forall t>0$. Let the observer gains be chosen such that

$$
\begin{aligned}
K_{v_{y}} & >0 \\
k_{x} & >\frac{1}{4} \frac{K_{v_{y}} c_{3}^{2}}{c_{1}} \\
K_{r} & >c_{5}+\frac{c_{7}}{4 k_{x} K_{v_{y}} c_{1}-\left(K_{v_{y}} c_{3}\right)^{2}}
\end{aligned}
$$

where

$$
\begin{aligned}
& c_{7}=\left(K_{v_{y}} c_{2}+c_{4}\right)\left(k_{x}\left(K_{v_{y}} c_{2}+c_{4}\right)-\frac{1}{2} K_{v_{y}} c_{3} c_{6}\right)+ \\
& c_{6}\left(\frac{1}{2} K_{v_{y}} c_{3}\left(K_{v_{y}} c_{2}+c_{4}\right)+K_{v_{y}} c_{1} c_{6}\right) .
\end{aligned}
$$

Then, if $\|\tilde{\mathbf{x}}(0)\| \leq \rho$, the state $\hat{\mathbf{x}}(t)$ of the observer (5) converges to the state $\mathbf{x}(t)$ of the system (4), and the origin of the observer error dynamics (8) is uniformly exponentially stable.

PRoOF Define the Lyapunov function candidate $V(\tilde{\mathbf{x}})=$ $\frac{1}{2}\left(\tilde{v}_{x}^{2}+\tilde{v}_{y}^{2}+\tilde{r}^{2}\right)$. The time derivative along the trajectories of the error dynamics (8) is

$$
\begin{aligned}
& \dot{V}=\tilde{v}_{x}\left(\tilde{v}_{y} r-\sum_{i=1}^{4} K_{i}\left(a_{x}\right) \tilde{v}_{x}\right) \\
& +\tilde{v}_{y}\left(-\tilde{v}_{x} r+K_{v_{y}} \sum_{i=1}^{4}\left[\begin{array}{ll}
0 & 1]
\end{array}\right] \mathbf{R}\left(\delta_{i}\right)\left(\mathbf{F}_{i}-\hat{\mathbf{F}}_{i}\right)\right) \\
& +\tilde{r}\left(\frac{1}{J_{z}} \sum_{i=1}^{4} \mathbf{g}_{i}^{\top} \mathbf{R}\left(\delta_{i}\right)\left(\mathbf{F}_{i}-\hat{\mathbf{F}}_{i}\right)-K_{r} \tilde{r}\right) .
\end{aligned}
$$

Assuming the conditions for Lemma 1 holds, this can be upper bounded:

$$
\begin{aligned}
& \dot{V} \leq-k_{x} \tilde{v}_{x}^{2}-K_{v_{y}} c_{1} \tilde{v}_{y}^{2}+K_{v_{y}} c_{2}|\tilde{r}|\left|\tilde{v}_{y}\right|+K_{v_{y}} c_{3}\left|\tilde{v}_{x}\right|\left|\tilde{v}_{y}\right| \\
&+c_{4} \tilde{r}\left|\tilde{v}_{y}\right|+c_{5} \tilde{r}|\tilde{r}|+c_{6} \tilde{r}\left|\tilde{v}_{x}\right|-K_{r} \tilde{r}^{2} \\
&=-|\tilde{\mathbf{x}}|^{\top} A|\tilde{\mathbf{x}}|,
\end{aligned}
$$

where $|\tilde{\mathbf{x}}|$ and the matrix $A$ are defined as

$$
|\tilde{\mathbf{x}}|:=\left(\begin{array}{c}
\left|\tilde{v}_{x}\right| \\
\left|\tilde{v}_{y}\right| \\
|\tilde{r}|
\end{array}\right), A:=\left(\begin{array}{ccc}
k_{x} & -\frac{1}{2} K_{v_{y}} c_{3} & -\frac{1}{2} c_{6} \\
-\frac{1}{2} K_{v_{y}} c_{3} & K_{v_{y}} c_{1} & -\frac{1}{2} K_{v_{y}} c_{2}-\frac{1}{2} c_{4} \\
-\frac{1}{2} c_{6} & -\frac{1}{2} K_{v_{y}} c_{2}-\frac{1}{2} c_{4} & K_{r}-c_{5}
\end{array}\right) .
$$

To show that $\dot{V}<-\kappa\|\tilde{\mathbf{x}}\|^{2}$ for some positive $\kappa$, we show how the conditions (9)-(11) imply that all principal minors of $A$ are positive, and thus that $A$ is positive definite.

The second principal minor is $k_{x} K_{v_{y}} c_{1}-\frac{1}{4}\left(K_{v_{y}} c_{3}\right)^{2}$. We see that due to (9), this is positive due to (10), which also ensures the positivity of the first principal minor $\left(k_{x}\right)$. The third principal minor (the determinant of $A$ ) is (developed after the third column)

$$
\begin{aligned}
\left(K_{r}-c_{5}\right)\left(k_{x}\right. & \left.K_{v_{y}} c_{1}-\frac{1}{4}\left(K_{v_{y}} c_{3}\right)^{2}\right) \\
& +\frac{1}{2}\left(K_{v_{y}} c_{2}+c_{4}\right) \operatorname{det} A_{2,3}-\frac{1}{2} c_{6} \operatorname{det} A_{1,3}
\end{aligned}
$$


where $A_{i, j}$ is the $2 \times 2$ matrix produced by removing row $i$ and column $j$ from $A$. Noting that $\operatorname{det} A_{2,3}$ and $\operatorname{det} A_{1,3}$ do not depend on $K_{r}$, we see that this determinant can be made positive by choosing $K_{r}$ large enough. Doing the tedious calculations, we end up with the bound (11).

By assumption, $\mathbf{x}(t) \in X_{s}(\rho, \boldsymbol{\delta}(t), \boldsymbol{\omega}(t)) \subset X(\boldsymbol{\delta}(t), \boldsymbol{\omega}(t))$ $\forall t>0$, and we must show that $\hat{\mathbf{x}}(t) \in X(\boldsymbol{\delta}(t), \boldsymbol{\omega}(t))$ $\forall t>0$ such that the conditions for Lemma 1 holds. Since $\mathbf{x}(t) \in X_{s}(\rho ; \boldsymbol{\delta}(t), \boldsymbol{\omega}(t))$ and $\|\tilde{\mathbf{x}}(0)\| \leq \rho, \hat{\mathbf{x}}(0) \in$ $X(\boldsymbol{\delta}(0), \boldsymbol{\omega}(0))$. From the above, $\frac{d}{d t}\|\tilde{\mathbf{x}}\|=\frac{\dot{V}}{\|\tilde{\mathbf{x}}\|}<0$, which means that $\|\tilde{\mathbf{x}}(t)\| \leq \rho \forall t>0$, and thus $\hat{\mathbf{x}}(t)$ will remain in $X(\boldsymbol{\delta}(t), \boldsymbol{\omega}(t))$. Thus, from $\dot{V}<-\kappa\|\tilde{\mathbf{x}}\|^{2}$ and standard Lyapunov theory [8], we conclude that $\tilde{\mathbf{x}}(t) \rightarrow 0$ with exponential convergence rate.

The same Lyapunov function will be used to prove the ISS properties. A non-zero $u(t)$ gives (for small $\tilde{\mathbf{x}}$ )

$$
\dot{V} \leq-\lambda_{\min }(A)\|\tilde{\mathbf{x}}\|^{2}+\|\tilde{\mathbf{x}}\||u|
$$

which shows that

$$
\dot{V} \leq-\frac{\lambda_{\min }(A)}{2}\|\tilde{\mathbf{x}}\|^{2}, \quad \forall\|\tilde{\mathbf{x}}\| \geq \frac{2}{\lambda_{\min }(A)}|u|
$$

implying

$$
\begin{aligned}
\|\tilde{\mathbf{x}}(t)\| \leq\left\|\tilde{\mathbf{x}}\left(t_{0}\right)\right\| e^{-\frac{\lambda_{\min }(A)}{2}\left(t-t_{0}\right)} & \\
& +\frac{2}{\lambda_{\min }(A)}\left(\sup _{t_{o} \leq \tau \leq t}|u(\tau)|\right)
\end{aligned}
$$

assuming the supremum exists. Since the conditions in Theorem 1 do not hold globally, we conclude a local variant of ISS:

Corollary 1 Assume that the conditions of Theorem 1 holds for all $(\boldsymbol{\delta}, \boldsymbol{\omega}) \in \Delta \times \Omega$ on $\|\tilde{\mathbf{x}}\| \leq d_{x}$ and $|u| \leq d_{u}$. Then there exists positive constants $k_{1}$ and $k_{2}$ such that (12) holds for $\left\|\tilde{\mathbf{x}}\left(t_{0}\right)\right\|<k_{1}$ and $\sup _{t>t_{0}}|u(t)|<k_{2}$.

PROOF Follows from local ISS [8, p. 192] and the above. Since the conditions of Theorem 1 typically hold in a large operating region ("large" $d_{x}$ and $d_{u}$ can be found), it follows that $k_{1}$ and $k_{2}$ are not necessarily "small".

\section{OBSERVER ROBUSTNESS}

The previous analysis have been based on the assumption of no model-plant mismatch, that is, that the friction model used in the observer represents the real relation between wheel slips and friction forces. In this section we will first show that the observer error dynamics is ISS also with respect to errors in the friction model. Furthermore, assuming Assumption 2 holds globally for the friction model used in the observer, we will show that observer error (and thus the states of the observer) will stay bounded for any initial conditions regardless of modelplant mismatches, as long as the real vehicle states and measurements are bounded.

Assume that the observer friction model $\left(\mathbf{F}_{i}\right)$ is related to the real relation between wheel slips and wheel forces (the "real" friction model, $\mathbf{F}_{r, i}$ ) in the following way:

$$
\mathbf{F}_{i}\left(\mathbf{x}, \delta_{i}, \omega_{i}\right)=\mathbf{F}_{r, i}\left(\mathbf{x}, \delta_{i}, \omega_{i}\right)+\boldsymbol{\Delta} \mathbf{F}_{i}\left(\mathbf{x}, \delta_{i}, \omega_{i}\right)
$$

where $\Delta \mathbf{F}$ represents the model-plant mismatch. The difference between the real forces and the observer forces can then be written

$$
\begin{aligned}
\mathbf{F}_{r, i}\left(\mathbf{x}, \delta_{i}, \omega_{i}\right)-\mathbf{F}_{i}\left(\hat{\mathbf{x}}, \delta_{i}, \omega_{i}\right) \\
\quad=\mathbf{F}_{i}\left(\mathbf{x}, \delta_{i}, \omega_{i}\right)-\mathbf{F}_{i}\left(\hat{\mathbf{x}}, \delta_{i}, \omega_{i}\right)-\boldsymbol{\Delta} \mathbf{F}_{i}\left(\mathbf{x}, \delta_{i}, \omega_{i}\right),
\end{aligned}
$$

which, when inserted in (8), gives the following observer error system:

$$
\begin{aligned}
& \dot{\tilde{v}}_{x}=\tilde{v}_{y} r-\sum_{i=1}^{4} K_{i}\left(a_{x}, \boldsymbol{\omega}\right) \tilde{v}_{x}+u_{1} \\
& \dot{\tilde{v}}_{y}=-\tilde{v}_{x} r+K_{v_{y}} \sum_{i=1}^{4}\left[\begin{array}{ll}
0 & 1
\end{array}\right] \mathbf{R}\left(\delta_{i}\right)\left(\mathbf{F}_{i}-\hat{\mathbf{F}}_{i}\right)+u_{2} \\
& \dot{\tilde{r}}=\frac{1}{J_{z}} \sum_{i=1}^{4} \mathbf{g}_{i}^{\top} \mathbf{R}\left(\delta_{i}\right)\left(\mathbf{F}_{i}-\hat{\mathbf{F}}_{i}\right)-K_{r} \tilde{r}+u_{3}
\end{aligned}
$$

where $u_{1}$ is as defined in (7), and

$$
\begin{aligned}
& u_{2}=-K_{v_{y}} \sum_{i=1}^{4}\left[\begin{array}{ll}
0 & 1
\end{array}\right] \mathbf{R}\left(\delta_{i}\right) \Delta \mathbf{F}_{i}\left(\mathbf{x}, \delta_{i}, \omega_{i}\right), \\
& u_{3}=-\frac{1}{J_{z}} \sum_{i=1}^{4} \mathbf{g}_{i}^{\top} \mathbf{R}\left(\delta_{i}\right) \Delta \mathbf{F}_{i}\left(\mathbf{x}, \delta_{i}, \omega_{i}\right) \text {. }
\end{aligned}
$$

Let $\mathbf{u}=\left(u_{1}, u_{2}, u_{3}\right)^{\top}$. Note that Theorem 1 shows exponential stability when $\mathbf{u}=0$. For $\mathbf{u} \neq 0$, a similar procedure as the one leading to Corollary 1 can be used. Under the assumptions of Theorem 1 ,

$$
\dot{V} \leq-\lambda_{\min }(A)\|\tilde{\mathbf{x}}\|^{2}+\|\tilde{\mathbf{x}}\|\|\mathbf{u}\|
$$

implying

$$
\begin{aligned}
\|\tilde{\mathbf{x}}(t)\| \leq\left\|\tilde{\mathbf{x}}\left(t_{0}\right)\right\| e^{-\frac{\lambda_{\min }(A)}{2}\left(t-t_{0}\right)} & \\
& +\frac{2}{\lambda_{\min }(A)}\left(\sup _{t_{o} \leq \tau \leq t}\|\mathbf{u}(\tau)\|\right)
\end{aligned}
$$

assuming the supremum exists. The following local ISS result follows:

Corollary 2 Assume that the conditions of Theorem 1 holds for all $(\boldsymbol{\delta}, \boldsymbol{\omega}) \in \Delta \times \Omega$ on $\|\tilde{\mathbf{x}}\| \leq d_{x}$ and $\|\mathbf{u}\| \leq$ $d_{u}$. Then there exists positive constants $k_{1}$ and $k_{2}$ such that (14) holds for $\left\|\tilde{\mathbf{x}}\left(t_{0}\right)\right\|<k_{1}$ and $\sup _{t>t_{0}}\|\mathbf{u}(t)\|<k_{2}$.

When $\mathbf{u}=0$ in (13), then the remaining error system depend only on the friction model used in the observer. If one makes sure that this friction model fulfills that

- Assumption 2 holds globally (for this, one must take care with singularities in slip definitions), 
- The friction model errors $\Delta \mathbf{F}_{i}$ are bounded (since the real friction forces are bounded, this is trivial to obtain),

then we can conclude that the observer states are globally bounded:

Corollary 3 Assume Assumption 2 holds for all $\hat{\mathrm{x}}$ $\left(X(\boldsymbol{\delta}, \boldsymbol{\omega})=\mathbb{R}^{n}\right)$ for the friction model used in the observer, and that the observer gains are chosen according to (9)(11). Furthermore, assume that $\mathbf{u}$ is bounded. Then the observer error is (uniformly) globally bounded.

PRoof Since Assumption 2 holds for all $\hat{x} \in \mathbb{R}^{n}$, Theorem 1 shows global exponential stability of the error dynamics when $\mathbf{u}=0$. In the general case $\mathbf{u} \neq 0$, for which (14) now holds globally. Thus boundedness of $\mathbf{u}$ implies boundedness of $\tilde{\mathbf{x}}$.

This result clearly implies that if the real vehicle states are bounded, then (under the assumptions of Corollary 3 ) the observer states will always be bounded.

Remark 2 Corollary 3 requires boundedness of $u_{i}$. At first sight, it might appear that $u_{1}=u$ as defined in (7) will become unbounded when $\alpha_{i}= \pm \pi / 2$ (it is easily seen that $\lambda_{i, x}=-1$ is not a real singularity). We have that $\alpha_{i} \rightarrow \pi / 2$ only when $V_{i, x} \rightarrow 0$, and on closer inspection the limit $\lim _{V_{i, x} \rightarrow 0} u$ is bounded (actually, the limit as stated is undefined, but the one-sided limits are both defined and bounded). However, this is purely technicalities, the purpose of the term $u$ is to capture the difference between the real longitudinal velocity and the longitudinal velocities calculated by (6). When $V_{i, x}=0$ this will clearly be bounded as long as (the measurements) $\omega_{i}$ are bounded.

\section{OBSERVER TUNING}

The stability conditions (9)-(11) of Theorem 1 provide a starting point for tuning the gains of the observer.

$K_{i}\left(a_{x}, \boldsymbol{\omega}\right)$ : The condition (10) says that the sum of these should be sufficiently positive. In addition, it is advisable to let them be time-varying: Since they decide the influence of the wheel speed measurements, they should be low in conditions with large longitudinal wheel slips, and high when longitudinal wheel slips are low.

For instance, in the case of positive acceleration, driven wheels will have higher slips than non-driven wheels. In $[10,6]$ tuning rules based on similar considerations using the $a_{x}$ measurement are given. In addition, since the wheels in some cases might spin or block, it makes sense to also let $K_{i}$ be dependent on $\boldsymbol{\omega}$. This is implemented herein by letting $K_{i}$ be smaller the further away the present calculated wheel speed is from what we expect it to be (where the "expected" wheel speed is chosen judiciously).

$K_{v_{y}}$ : This gain weights the error in measured acceleration compared to calculated acceleration based on the friction model and estimated states. Since the acceleration sensor can give noisy data and a friction model might have varying quality, it is sensible to not tune this too high.

A very interesting tuning is $K_{v_{y}}=1 / \mathrm{m}$. This particular choice of $K_{v_{y}}$ makes $\dot{\hat{v}}_{y}$ (5b) independent of $a_{y}$, $\dot{\hat{v}}_{y}=-\hat{v}_{x} r+\frac{1}{m} \sum_{i=1}^{4}\left[\begin{array}{ll}0 & 1\end{array}\right] \mathbf{R}\left(\delta_{i}\right) \hat{\mathbf{F}}_{i}$, hence implying fault tolerance and robustness to noise in this measurement. At the same time, this is not a very aggressive (high) tuning, in the sense that it does not put very large weight on the friction model.

$K_{r}$ : Since the yaw-rate state is measured directly, this gain can be tuned more aggressively.

Lastly, it should be mentioned that signal processing to handle sensor issues such as sensor bias, is important for an successful implementation.

\section{DISCUSSION OF ASSUMPTION 2}

This section discusses Assumption 2 for two friction models. Most friction models can be written as a function of (lateral and longitudinal) tyre slips $\mathbf{F}_{i}=\mathbf{F}_{i}\left(\lambda_{i, x}, \lambda_{i, y}\right)$ only, and depend thus on vehicle velocity only indirectly. Therefore, we can write the partial derivatives

$$
\begin{aligned}
& \frac{\partial F_{i, x}}{\partial v_{y}}=\frac{\partial F_{i, x}}{\partial \lambda_{i, x}} \frac{\partial \lambda_{i, x}}{\partial v_{y}}+\frac{\partial F_{i, x}}{\partial \lambda_{i, y}} \frac{\partial \lambda_{i, y}}{\partial v_{y}}=\frac{\partial F_{i, x}}{\partial \lambda_{i, y}} \cos \alpha_{i} \frac{\partial \alpha_{i}}{\partial v_{y}} \\
& \frac{\partial F_{i, y}}{\partial v_{y}}=\frac{\partial F_{i, y}}{\partial \lambda_{i, x}} \frac{\partial \lambda_{i, x}}{\partial v_{y}}+\frac{\partial F_{i, y}}{\partial \lambda_{i, y}} \frac{\partial \lambda_{i, y}}{\partial v_{y}}=\frac{\partial F_{i, y}}{\partial \lambda_{i, y}} \cos \alpha_{i} \frac{\partial \alpha_{i}}{\partial v_{y}}
\end{aligned}
$$

where we have assumed $\frac{\partial \lambda_{i, x}}{\partial v_{y}}=0$. Since

$$
\frac{\partial \alpha_{i}}{\partial v_{y}}=\frac{-1}{1+\tan ^{2}\left(\delta_{i}-\alpha_{i}\right)}<0,
$$

we see that it is the lateral slip partial derivatives that determine if Assumption 2 holds (assuming $\alpha_{i}<\pi / 2$ ).

LINEAR FRICTION MODELS Linear friction models says that the friction forces are proportional to the slips,

$$
\left(\begin{array}{l}
F_{i, x}\left(\lambda_{x, i}, \lambda_{y, i}\right) \\
F_{i, y}\left(\lambda_{x, i}, \lambda_{y, i}\right)
\end{array}\right)=\left(\begin{array}{l}
C_{x} \lambda_{i, x} \\
C_{y} \lambda_{i, y}
\end{array}\right)
$$

where $C_{x}$ and $C_{y}$ are tyre (slip and cornering) stiffness coefficients. We conclude that Assumption 2 holds as discussed in Remark 1 , for some $\bar{\alpha}<\pi / 2$, since $C_{y}$ generally is positive.

THE MAGIC FORMULA TYRE MODEL The "magic formula tyre model" [12] is a widely used semi-empirical model for calculating steady-state tyre forces. The "combined slip" magic formula provides similar formulas for lateral and longitudinal tyre forces,

$$
\begin{aligned}
& F_{x}\left(\lambda_{x}, \lambda_{y}\right)=G_{x}\left(\lambda_{y}\right) F_{x 0}\left(\lambda_{x}\right), \\
& F_{y}\left(\lambda_{x}, \lambda_{y}\right)=G_{y}\left(\lambda_{x}\right) F_{y 0}\left(\lambda_{y}\right)
\end{aligned}
$$


where we have simplified somewhat since one of the parameters in $G_{x}\left(G_{y}\right)$ that according to [12] depends on $\lambda_{x}$ $\left(\lambda_{y}\right)$ is assumed constant. For notational convenience we drop the dependence on wheel index $i$ in this section.

The functions $F_{x 0}$ and $F_{y 0}$ are the "pure slip" formulas,

$$
F_{x 0}\left(\lambda_{x}\right)=D_{x} \sin \zeta_{x}, \quad F_{y 0}\left(\lambda_{y}\right)=D_{y} \sin \zeta_{y}
$$

where

$$
\begin{aligned}
& \zeta_{x}=C_{x} \arctan \left\{B_{x} \lambda_{x}-E_{x}\left(B_{x} \lambda_{x}-\arctan B_{x} \lambda_{x}\right)\right\} \\
& \zeta_{y}=C_{y} \arctan \left\{B_{y} \lambda_{y}-E_{y}\left(B_{y} \lambda_{y}-\arctan B_{y} \lambda_{y}\right)\right\} .
\end{aligned}
$$

The functions $G_{x}$ and $G_{y}$ are defined as $G_{x}\left(\lambda_{y}\right)=\cos \eta_{x}$ and $G_{y}\left(\lambda_{x}\right)=\cos \eta_{y}$ where

$\eta_{x}=C_{G x} \arctan \left\{B_{G x} \lambda_{y}-E_{G x}\left(B_{G x} \lambda_{y}-\arctan B_{G x} \lambda_{y}\right)\right\}$

$\eta_{y}=C_{G y} \arctan \left\{B_{G y} \lambda_{x}-E_{G y}\left(B_{G y} \lambda_{x}-\arctan B_{G y} \lambda_{x}\right)\right\}$.

We then have that

$$
\frac{\partial F_{y}}{\partial \lambda_{y}}=G_{y}\left(\lambda_{x}\right) \frac{B_{y} C_{y} D_{y}\left(1-E_{y}\left(1-\frac{1}{1+B_{y}^{2} \lambda_{y}^{2}}\right)\right) \cos \zeta_{y}}{1+\zeta_{y}^{2}} .
$$

Since $G\left(\lambda_{x}\right)>0$ and $E_{y} \leq 1$ [12, p. 189], $\frac{\partial F_{y}}{\partial \lambda_{y}}>0$ for $\zeta_{y}<\pi / 2$. For "shape factor" $C_{y}<1$, this holds for all $\lambda_{y}$. For $C_{y}>1$, the friction force declines for large $\lambda_{y} \mathrm{~s}$, and $\frac{\partial F_{y}}{\partial \lambda_{y}}>0$ only to the left of the peak of the friction curve, that is, for $\left|\lambda_{y}\right| \leq \bar{\lambda}$ where $\bar{\lambda}$ is defined by the solution to

$$
E_{y}=\frac{B_{y} \bar{\lambda}-\tan \frac{\pi}{2 C_{y}}}{B_{y} \bar{\lambda}-\arctan \left(B_{y} \bar{\lambda}\right)} .
$$

Furthermore,

$$
\frac{\partial F_{x}}{\partial \lambda_{y}}=-F_{x 0}\left(\lambda_{x}\right) \frac{B_{G x} C_{G x}\left(1-E_{G x}\left(1-\frac{1}{1+B_{G x}^{2} \lambda_{y}^{2}}\right)\right) \sin \eta_{x}}{1+\eta_{x}^{2}}
$$

We see that $\operatorname{sign} \frac{\partial F_{x}}{\partial \lambda_{y}}=-\operatorname{sign}\left(\lambda_{x} \lambda_{y}\right) \quad$ (since $\operatorname{sign} F_{x 0}\left(\lambda_{x}\right)=\operatorname{sign} \zeta_{x}=\operatorname{sign} \lambda_{x}$ and $\left.\operatorname{sign} \eta_{x}=\operatorname{sign} \lambda_{y}\right)$.

From the above, we make the following observations:

- For sufficiently small side-slip angles (that is, $\left|\lambda_{y}\right|<$ $\bar{\lambda}$ ) and small $\delta$, the first part of (2) is negative and dominates the second part.

- For $\lambda_{x} \approx 0$ the second part of (2) is approximately zero, and hence dominated by the first part.

- For large side-slip angles, the first part of (2) will get less negative, and even positive if $C_{y}>1$. However, in the case of braking $\left(\lambda_{x}<0\right)$, then the second part of (2) will often contribute in fulfilling the assumption: Since $\operatorname{sign} \frac{\partial F_{x}}{\partial v_{y}}=\operatorname{sign} \lambda_{x} \operatorname{sign} \alpha$, assuming that $\operatorname{sign} \alpha=\operatorname{sign} \delta$, gives $\frac{\partial F_{x}}{\partial v_{y}} \sin \delta<0$.
In conclusion, (2) is negative for realistic slip values and sufficiently small steering angles for tyres with $C_{y}<1$. For tyres with $C_{y}>1$, then for some combinations of $\lambda_{x}$ and (large) $\lambda_{y}$, it might be positive. Since it is the sum for all tyres that should be negative, a positive summand for one (or two) wheel(s) might be weighed against negative summands for the other wheels (for instance, rear wheels will often have lower side-slip angles due to small steering angle values).

When a friction model with computable partial derivatives is available, then (2) can be evaluated based on the estimates, either online or offline. Since (2) essentially is the only assumption that is required for the analysis herein, one could imagine using the value for convergence monitoring, somewhat akin to monitoring covariances in an EKF, and take corrective steps when the value becomes positive.

However, the above discussion shows that the only case when Assumption 2 might not hold for the friction model, is for large side-slip values, which physically can only remain for limited time intervals. As shown in Corollary 3, as long as at least the friction model used in the observer fulfills Assumption 2, the observer states will always remain bounded, also in these time intervals. Therefore it is not crucial if Assumption 2 does not hold for the real friction for all slip values.

The experimental validation in the next section indicates that for the friction model used in the experiments (which was not modified in any way for the observer), Assumption 2 was satisfied at all times in the test cases.

\section{EXPERIMENTAL RESULTS}

In this section, the observer is applied to experimental data from a car. The velocity estimates $\left(\hat{v}_{x}, \hat{v}_{y}\right.$, and side slip angle $\left.\hat{\beta}=\arctan \left(-\hat{v}_{y} / \hat{v}_{x}\right)\right)$ are compared to velocity measurements obtained using an optical sensor placed in front of the vehicle.

The gains of the observer are the same in all experiments. The gains in the longitudinal velocity observer vary between 0 and 200 (but such that $\sum_{i=0}^{4} K_{i}\left(a_{x}\right)>0$ always), and $K_{v_{y}}=1 / m$ and $K_{r}=20$.

Initial conditions for the observer are one of the wheel speeds (transformed to CG) for $\hat{v}_{x}, 0$ for $\hat{v}_{y}$ and 0 (or $r$ ) for $\hat{r}$. Experimenting with the initial conditions indicate a large region of attraction (as long as the choice of maximum friction coefficient $\mu_{H}$ in the friction model is reasonable).

Flat dry road, slalom maneuver The maximum friction coefficient used in the observer is set to $\mu_{H}=1$. The longitudinal velocity is shown in Fig. 2, while lateral veloc- 


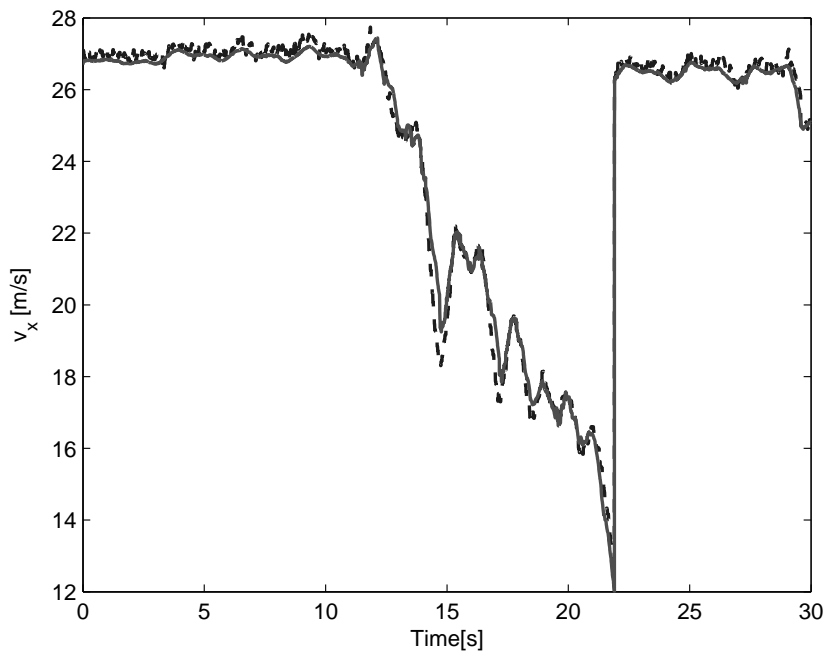

Figure 2: Estimate (solid) and measurement (dashed) of longitudinal velocity, slalom maneuver.

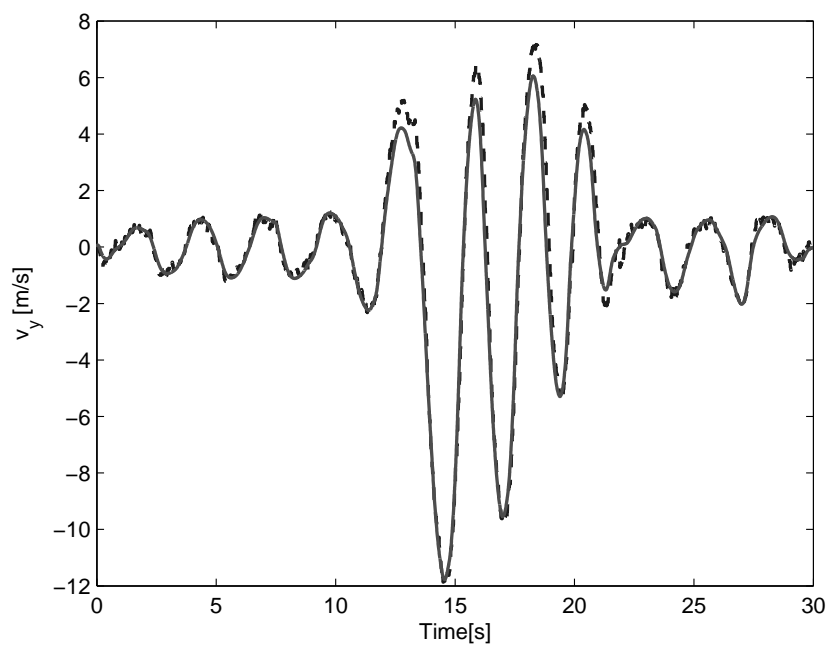

Figure 3: Estimate (solid) and measurement (dashed) of lateral velocity, slalom maneuver.

ity is shown in Fig. 3. The quality of both estimates are fine, so the vehicle side slip estimate, shown in Fig. 4, is also good.

Ice, driving in circle The maximum friction coefficient used in the observer is set to $\mu_{H}=0.3$. The longitudinal velocity is shown in Fig. 5 . The estimate is noisy, mainly due to large variations in the wheel speed measurements (caused by varying longitudinal slips).

The deviation in lateral velocity in this period is probably due to a combination of

- less accurate friction model for large lateral slip values, and (more likely)

- the maximal friction coefficient changes: Inspection

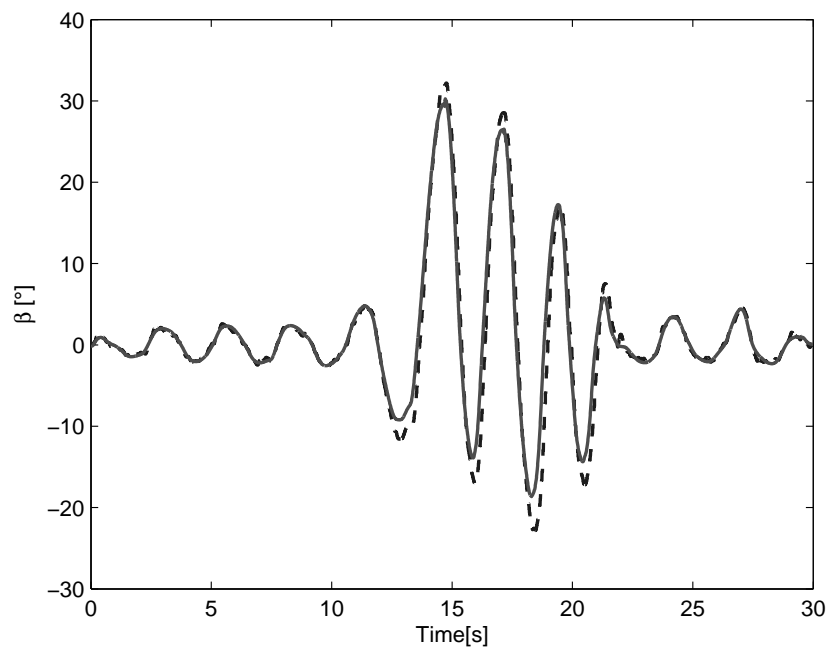

Figure 4: Estimate (solid) and measurement (dashed) of side slip, slalom maneuver.

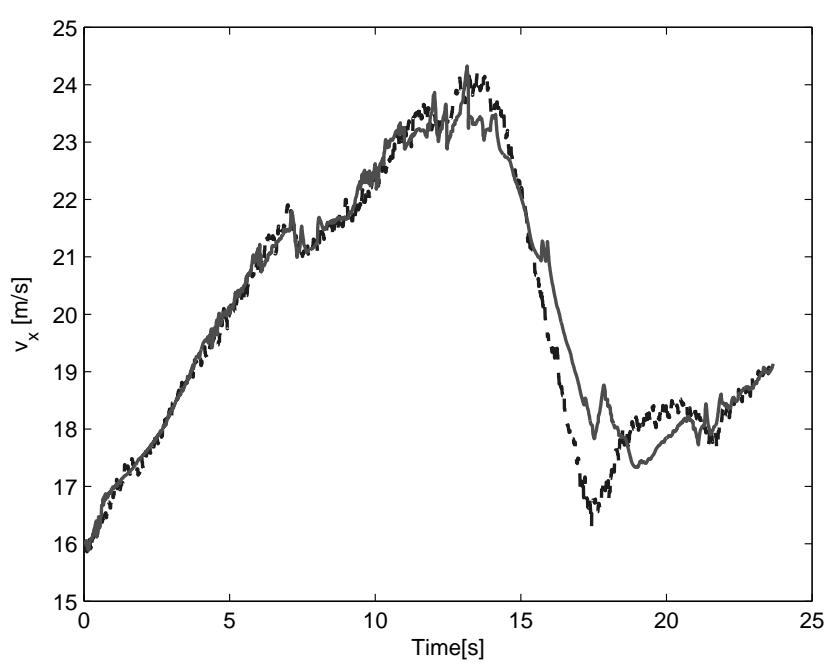

Figure 5: Estimate (solid) and measurement (dashed) of longitudinal velocity, circle on ice, full observer.

of the results indicates that the friction coefficient increases significantly after about 15 seconds, before it decreases again at around 18 seconds (in the same period, the ESP-flag is on). Setting the friction coefficient to 0.5 between 15 and 18 seconds gives significantly better estimates of lateral velocity (not shown here).

As a verification of the main convergence assumption (Assumption 2), the condition (2) is calculated on basis of the friction model, using the velocity estimates and our guess of the maximal friction coefficient. The results are plotted in Figure 8 for both time series, and we see that the condition is always satisfied in both cases. The friction model used, is an in-house friction model of similar complexity to the magic formula tyre model. 


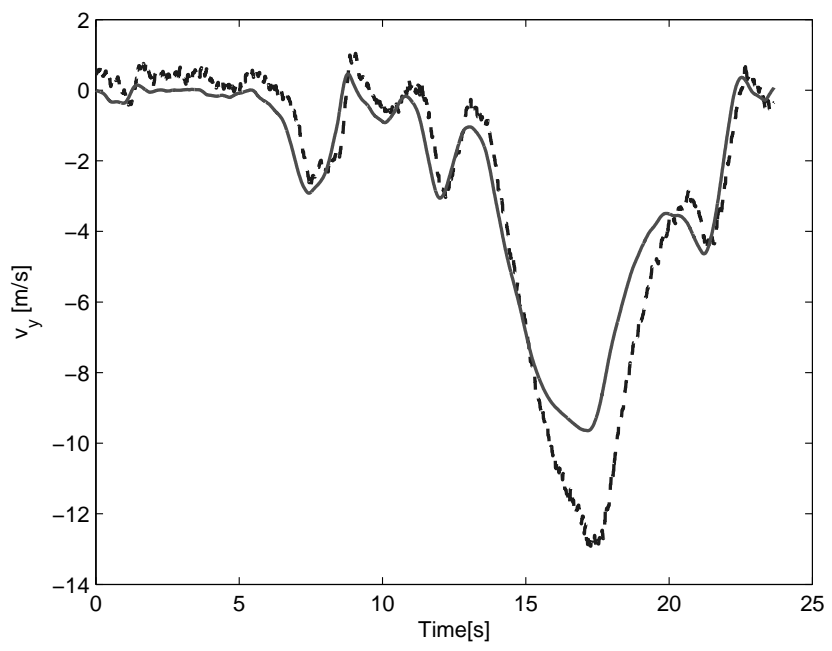

Figure 6: Estimate (solid) and measurement (dashed) of lateral velocity, circle on ice, full observer.

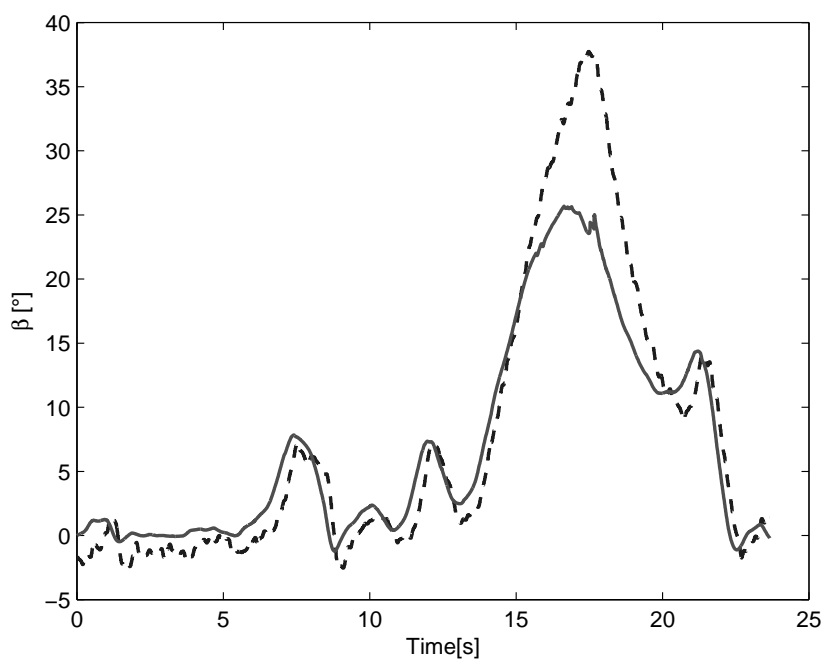

Figure 7: Estimate (solid) and measurement (dashed) of side slip, circle on ice, full observer.

\section{CONCLUSION}

A nonlinear observer for vehicle velocity and side-slip was proposed, which was proved to possess ISS-type stability properties under a certain condition on the friction model. The observer performs well when applied to experimental data from a car.

The main advantage of using nonlinear observers compared to an Extended Kalman Filter (EKF), is reduced computational complexity. While the number of ODEs to solve for an EKF is $3 / 2 n+1 / 2 n^{2}$, nonlinear observers usually have to solve $n$ (the number of estimated variables) ODEs. In addition, for the EKF the nonlinear ODE (and/or measurement equation) have to be linearized at each sample, which along with monitoring of boundedness of the covariance matrix estimate also might induce considerable computation. The few tuning knobs of the
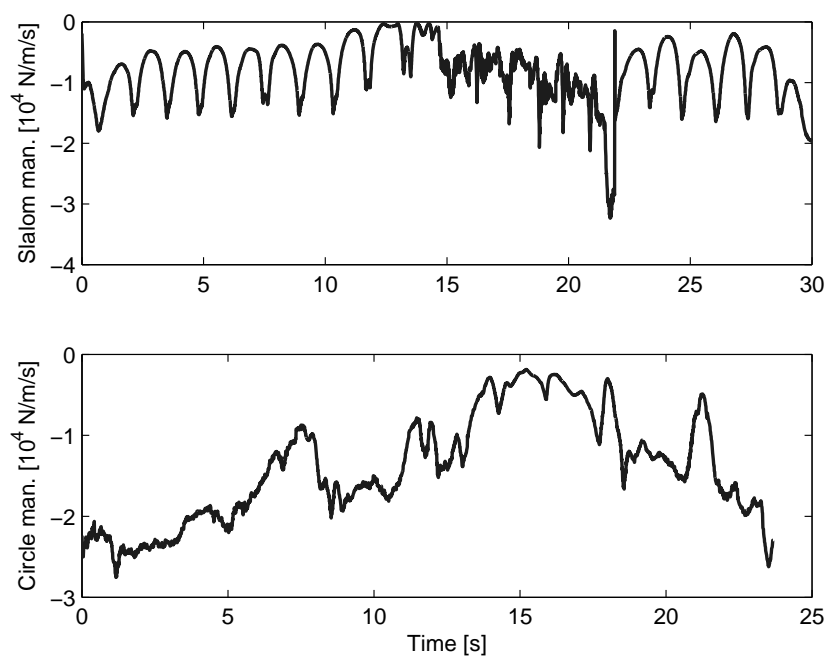

Figure 8: Condition (2) for slalom maneuver on asphalt, and circle maneuver on ice. The curves verify that Assumption 2 is always satisfied in these test drives.

observer simplifies tuning compared to tuning the covariance matrices of an EKF.

A requirement for good performance of the nonlinear observer, is information on the maximal friction coefficient between tyre and road. Information on road bank angle, and possibly inclination angle will be important in the case of a non-flat road.

\section{ACKNOWLEDGMENTS}

This research is supported by the European Commission STREP project CEMACS, contract 004175.

\section{REFERENCES}

[1] M. C. Best, T. J. Gordon, and P. J. Dixon. An extended adaptive Kalman filter for real-time state estimation of vehicle handling dynamics. Vehicle System Dynamics, 34:57-75, 2000.

[2] J. Farrelly and P. Wellstead. Estimation of vehicle lateral velocity. In Proc. 35th IEEE Conf. Decision Contr., pages 552-557, 1996.

[3] Y. Fukada. Slip-angle estimation for stability control. Vehicle Systems Dynamics, 32:375-388, 1999.

[4] A. Hac and M. D. Simpson. Estimation of vehicle side slip angle and yaw rate. In SAE 2000 World Congress, Detroit, MI, USA, 2000.

[5] M. Hiemer, A. von Vietinghoff, U. Kiencke, and T. Matsunaga. Determination of vehicle body slip angle with non-linear observer strategies. In Proceedings of the SAE World Congress, 2005. Paper no. 2005-01-0400. 
[6] L. Imsland, T. A. Johansen, T. I. Fossen, J. Kalkkuhl, and A. Suissa. Vehicle velocity estimation using modular nonlinear observers. In Proc. 41th IEEE Conf. Decision Contr., 2005.

[7] J. Kalkkuhl, T. A. Johansen, and J. Ludemann. Nonlinear adaptive backstepping with estimator resetting using multiple observers. In R. Johansson and A. Rantzer, editors, Nonlinear and Hybrid Systems in Automotive Control. Springer Verlag, 2003.

[8] H. K. Khalil. Nonlinear Systems. Prentice Hall, Upper Saddle River, NJ, 3rd edition, 2002.

[9] U. Kiencke and A. Daiss. Observation of lateral vehicle dynamics. Control Engineering Practice, 5(8):1145-1150, 1997.

[10] U. Kiencke and L. Nielsen. Automotive Control Systems. Springer, 2000.

[11] J. Lu and T. A. Brown. Vehicle side slip angle estimation using dynamic blending and considering vehicle attitude information. Patent US 6671595, 2003.

[12] H. B. Pacejka. Tyre and vehicle dynamics. Butterworth-Heinemann, 2002.

[13] L. R. Ray. Nonlinear state and tire force estimation for advanced vehicle control. IEEE Transactions on Control Systems Technology, 3(1):117-124, 1995.

[14] L. R. Ray. Nonlinear tire force estimation and road friction identification: simulation and experiments. Automatica, 33(10):1819-1833, 1997.

[15] E. D. Sontag and Y. Wang. On characterizations of the input-to-state stability property. Systems Control Lett., 24(5):351-359, 1995.

[16] A. Suissa, Z. Zomotor, and F. Böttiger. Method for determining variables characterizing vehicle handling. Patent US 5557520, 1996.

[17] A. Y. Ungoren and H. Peng. A study on lateral speed estimation methods. Int. J. Vehicle Autonomous Systems, 2(1/2):126-144, 2004.

[18] A. T. van Zanten. Bosch ESP system: 5 years of experience. In In Proceedings of the Automotive Dynamics \& Stability Conference (P-354), 2000. Paper no. 2000-01-1633.

[19] P. J. T. Venhovens and K. Naab. Vehicle dynamics estimation using Kalman filters. Vehicle System Dynamics, 32:171-184, 1999. 